\title{
Extracting Data Parallelism in Non-Stencil Kernel Computing by Optimally Coloring Folded Memory Conflict Graph
}

\author{
Juan Escobedo and Mingjie Lin \\ University of Central Florida \\ Department of Electrical and Computer Engineering \\ Orlando, FL, U.S.A.
}

\begin{abstract}
Irregular memory access pattern in non-stencil kernel computing renders the well-known hyperplane- [1], lattice- [2], or tessellationbased [3] HLS techniques ineffective. We develop an elegant yet effective technique that synthesizes memory-optimal architecture from high level software code in order to maximize applicationspecific data parallelism. Our basic idea is to exploit graph structures embedded in data access pattern and computation structure in order to perform the memory banking that maximizes parallel memory accesses while conserving both hardware and energy consumption. Specifically, we priority color a weighted conflict graph generated from folding the fundamental conflict graph to maximize memory conflict reduction. Most interestingly, our graph-based methodology enables a straightforward tradeoff between the number of memory banks and minimizing memory conflicts.

We empirically test our methodology with Vivado HLx 2015.4 on a standard Kintex-7 device for six benchmark computing kernels by measuring conflict reduction. In particular, our approach only require $9.56 \%$ LUT, $3.2 \% \mathrm{FF}, 2.5 \% \mathrm{BRAM}$, and $11.33 \%$ DSP of the total available hardware resource to obtain a mapping function that achieves a $90 \%$ conflict reduction on a modified forward Gaussian elimination Kernel with 4 simultaneous memory accesses.
\end{abstract}

\section{KEYWORDS}

Graph Folding, Graph Coloring, Memory Conflict Reduction

\section{ACM Reference Format:}

Juan Escobedo and Mingjie Lin. 2018. Extracting Data Parallelism in NonStencil Kernel Computing by Optimally Coloring Folded Memory Conflict Graph. In DAC '18: DAC '18: The 55th Annual Design Automation Conference 2018, June 24-29, 2018, San Francisco, CA, USA. ACM, New York, NY, USA, 6 pages. https://doi.org/10.1145/3195970.3196088

\section{INTRODUCTION}

Reconfigurable logic devices such as FPGAs offer a high degree of flexibility and multi-functionality for memory-intensive embedded applications with reduced turn-around time. This is mainly

Permission to make digital or hard copies of all or part of this work for personal or classroom use is granted without fee provided that copies are not made or distributed for profit or commercial advantage and that copies bear this notice and the full citation on the first page. Copyrights for components of this work owned by others than ACM must be honored. Abstracting with credit is permitted. To copy otherwise, or republish, to post on servers or to redistribute to lists, requires prior specific permission and/or a fee. Request permissions from permissions@acm.org.

DAC '18, Fune 24-29, 2018, San Francisco, CA, USA

(C) 2018 Association for Computing Machinery.

ACM ISBN 978-1-4503-5700-5/18/06 ..\$15.00

https://doi.org/10.1145/3195970.3196088 because that modern FPGA devices typically contain a large number of individual memory blocks which, if configured as independent memory banks and accessed in parallel, can offer tremendous aggregated memory access bandwidth while avoiding memory conflicts that cause prolonged memory access latency and potentially serious performance degradation. Recognizing this opportunity, many recent advances in FPGA High-Level Synthesis (HLS) have been made to optimize stencil-like memory access patterns with static offsets and achieved significant successes [1-5]. Unfortunately, in many irregular scientific codes, much more general memory access patterns exist and pose much greater challenges to achieving effective memory partitioning and mapping in order to faciltate parallel memory accesses. As such, in non-stencil kernel computing, finding a versatile yet cost-effective method to synthesize application-specific hardware module from high level software code to fully exploit memory-level parallelism remains a key challenge to overcome.
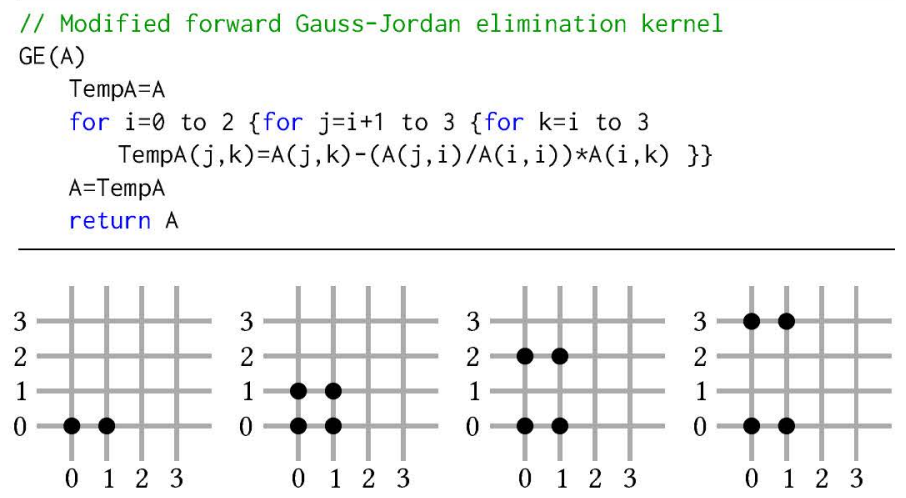

$(i, j, k)=(0,1,0) \quad(i, j, k) \overline{\overline{\mathbf{d}}}(0,1,1) \quad(i, j, k)=(0,1,2) \quad(i, j, k)=(0,1,3)$ Figure 1: (a) Modifie $\overline{\bar{d}}$ forward Gauss-Jordan elimination kernel. Accessed elements on iteration $(b)(i, j, k)=(0,1,1),(c)$ $(\mathbf{i}, \mathbf{j}, \mathbf{k})=(\mathbf{0}, \mathbf{1}, 2),(\mathrm{d})(\mathbf{i}, \mathbf{j}, \mathbf{k})=(\mathbf{0}, 2,2)$.

In scientific computation or signal processing applications, the most computationally intensive code segment often consists of iterative kernels that update array elements according to some specific pattern. This paper focuses on only non-stencil kernel computing, where kernel memory access patterns are changing dynamically across iterations. For example, in computational fluid dynamics (CFD), solving a modified forward Gauss-Jordan elimination kernel (Fig. 1(a)) will perform a sequence of sweeps (called time-steps) through a given array. As shown in Fig. 1(b), each iteration of this kernel will access a different node neighborhood. This is in contrast to the stencil-type kernels like Sobel or Denoise, where all these relative distances remain constant throughout its iterations. As such, the memory access pattern of non-stencil kernel computing is irregular. If still adopting conventional memory banking 
schemes, simultaneous accesses are often made to the same memory bank by many requests, consequently queuing many memory requests that cause longer memory latency. Even with methods such as [6], which could in theory tackle non-stencil problem, as the problem size grows, its resulting number of required memory banks for parallel memory access increases as well. Therefore, for arbitrarily large problems, the traditional approach yields an infeasible number of partitions. Clearly, exploiting the data parallelism offered by multi-bank memory architectures is crucial to improving the overall computing performance, which highly depends on effectively minimizing memory conflicts through efficient data distribution schemes.

\section{Research Objective and Contributions}

In this paper, we focus on the problem of distributing data accessed in a non-stencil computing kernel onto multiple independent memory banks such that a high degree of data-parallelism can be achieved Assuming that the HDL-synthesized computing structure constantly request multiple data items from the on-chip memory, our goal is to store data items among individual memory banks in such a manner that they can be accessed with as few (ideally, zero) conflicts as possible. Specifically, 1) we introduce a practical way to encode the conflict information from an arbitrarily large problem to a small, more manageable size; 2) We propose a weighted graph coloring algorithm to obtain a mapping function that suits the characteristics of the problem at hand and present an easy and intuitive way to calculate the memory bank index and intra bank offset. Most interestingly, the stencil-based memory banking problem, previously solved by a hyperplane-based methodology, can also be optimally solved as a special example with our proposed graph folding method.

\section{PROBLEM FORMULATION AND OVERALL SOLVING STRATEGY}

The central task of high-level synthesis is to transform a C-like software code segment into efficient and high-performance hardware circuit description. Typically, within a given code segment, the largest percentage of computation will be concentrated on iteratively executing a small-size computing kernel, which often access multiple data items stored in one or several data arrays as shown in Fig. 1. Mathematically, during each iteration, all the memory accesses within such a kernel $K$ can be defined as a set of $m$ data points $P=\left\{\overrightarrow{A_{0}}, \overrightarrow{A_{1}}, \ldots, \overrightarrow{A_{m-1}}\right\}$, where each data point $\vec{A}$. is stored in a $d$-dimensional array structure. The main objective of memory banking is to distribute all array elements into multiple independent memory banks such that fully parallel memory accesses can be enabled. In other words, during any iteration $i$, all accessed memory points in $P$ will be read from totally independent memory banks with zero memory reading conflicts. Mathematically, the memory banking problem of an $d$-dimensional array can be defined as finding a pair of mapping functions $f(\vec{x})$ and $g(\vec{x})$, where $f(\vec{x})$ assigns a distinct memory bank and $g(\vec{x})$ generates its corresponding intra-bank offset for a given data element, respectively. Clearly, an access conflict between two memory references $\vec{x}_{j}$ and $\vec{x}_{k}$ occurs if $f\left(\vec{x}_{j}\right)=f\left(\vec{x}_{k}\right)$, which means accessing the same memory bank during the same iteration. Again, we assume singleport memory banks here. As such, the memory banking problem under our consideration consists of two mapping problems: memory bank mapping and intra-bank offset mapping. The central objective of solving these two mapping problems is to minimize both the number of memory banks and the total memory usage.

Theoretically, one can optimally solve the memory banking problem by optimally coloring the complete memory access conflict graph. Here, a memory access conflict graph is generated by considering all accessed memory locations as nodes and adding edges between nodes that need to be accessed together, forming a clique, during a particular iteration for all iterations in the iteration domain. In other words, whenever two array elements $M_{i}$ and $M_{j}$ are accessed during the same iteration, we consider them are conflicting and need to allocated into two different memory banks. Here, we assume all memory banks to be single-ported for simplicity. After solving the optimal single-port memory banking problem, it can be readily shown that multiple-port memory banking can be solved by defective coloring scheme with the same strategy.

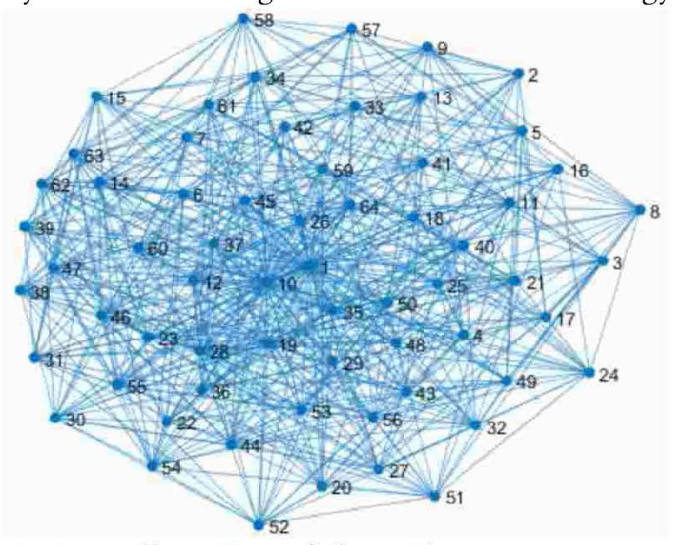

Figure 2: A small portion of the entire memory access conflict graph generated by the non-stencil kernel in Fig. 1.

The biggest advantage of such graph-coloring-based approach is its generality, i.e., this method imposes no limitation on the regularity of loop structure or the affiness of memory accesses. Unfortunately, in practice, this graph-based methodology is infeasible due to two critical issues. First, for any realistic code segment of loop, the size the memory access graph quickly increases with its associated array size, therefore simply too big for any existing graph coloring algorithm to handle optimally. For example, for the 4-point non-stencil $S$ shown in Fig. 1(b), even a tiny portion of its complete memory access conflict graph becomes quite complex as shown in Fig. 2, thus infeasible to optimally color. Second, even if we can optimally solve this graph coloring problem, the resulted memory address mapping will simply be too large, therefore completely impractical to implement with hardware. Unlike in stencilbased computing kernel, the repeatability of a complete memory access conflict graph can not be easily exploited. As such, optimally coloring the complete conflict graph can not be greatly reduced to optimally coloring a much smaller graph. The key challenge to developing our memory banking algorithm is how to exploit the special graph-theoretical property of our target memory access conflict graph while avoiding the NP-hardness of optimally coloring a large-sized complete memory access conflict graph. 
Figure 3: Flow diagram of our algorithm.

\section{Overall Methodology}

Fig. 3 presents the overall flow diagram of our graph-folding-based algorithm. Given a non-stencil computing kernel $S$, we first construct its associated conflict graph $G(S)$ and subsequently fold it into a much smaller weighted graph $F G(S)$. Next, $F G(S)$ will be priority colored with a given number of colors $k$. Here, $k$ directly corresponds to the number of independent single-port memory banks we choose to use. Clearly, $k$ will always less than $F G(S)$ 's chromatic number $\chi(F G(S))$. Finally, we complete our memory banking scheme by allocating each array element to a distinct memory bank denoted by a distinct color.

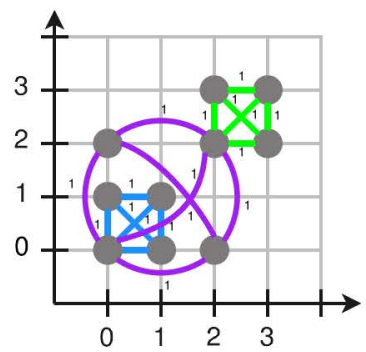

(a)

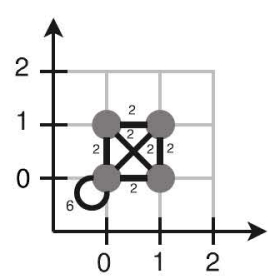

(c)

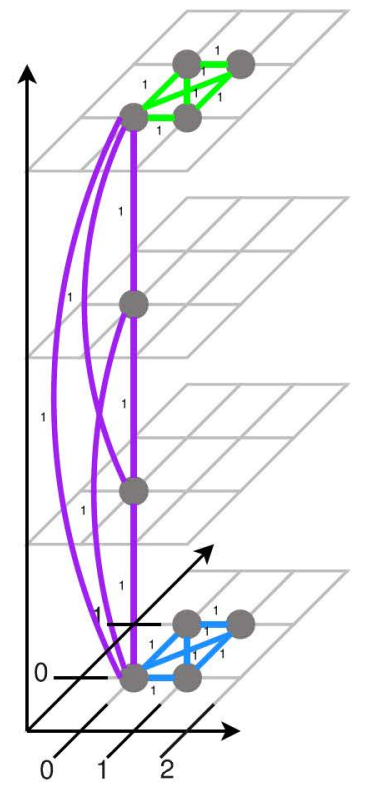

(b)

Figure 4: (a) Conflict graph for iterations $(i, j, k)=(0,1,1)$ in blue, $(i, j, k)=(0,2,2)$ in purple, and $(i, j, k)=(2,3,3)$ in green. $(b)$ Overlapping of $2 \times 2$ regions of the $4 \times 4$ problem. (c) Final weighted conflict graph for the iterations.

We now illustrate our strategy with a small $4 \times 4$ problem depicted in Fig. 1. If we fold the memory conflict graph in Fig. 4(a) two times, we obtain 4 subgraphs in Fig. 4(b), which can be collapsed into a weighted $2 \times 2$ graph in Fig. 4(c). We now priority-color this weighted graph with the detailed algorithm presented in Section 4. Depending on the maximum number of colors we use, we will be able to remove the majority of all conflicts. For example, in Fig. 4 , for the same $4 \times 4$ problem, 7 colors are needed to achieve truly conflict-free accesses. Interestingly, using merely 2, 3, and 4 colors, one would get 32,26 , and 20 conflicts, which corresponds to a reduction of $33.33 \%, 45.83 \%$, and $58.3 \%$ of the total conflicts, respectively.

Here, we define a conflict ratio $\gamma$ for a given conflict graph with a particular coloring scheme as the ratio between the number of memory conflicts with and without a memory banking scheme. Repeating this procedure for a problem of size $256 \times 256$ (for which a greedy graph coloring algorithm as the one presented in [7] gives a required number of colors of 259 for conflict free access), still with

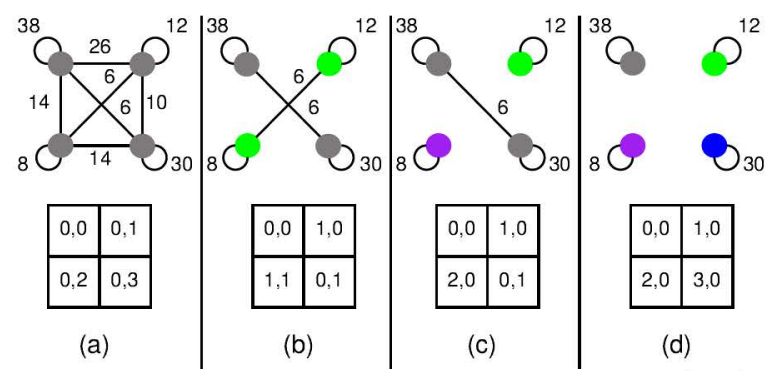

Figure 5: Coloring of the resulting conflict graph (top) and corresponding [bank, address] pair (bottom) for 1 bank (a), 2 banks (b), 3 banks (c), and 4 banks (d)

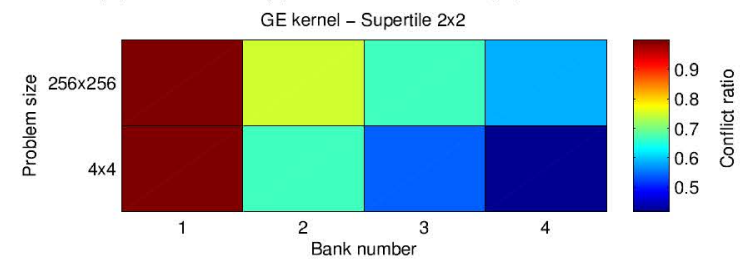

Figure 6: Conflict ratio for a folded graph size $2 \times 2$ on a problem of size $256 \times 256$ (top) and $4 \times 4$ (bottom) for $1,2,3$, and 4 banks

a folded graph of $2 \times 2$ and again using 2,3, and 4 colors, we get 191520,169664 , and 147808 conflicts respectively. This corresponds to a reduction of $25.1 \%, 33.46 \%$, and $41.83 \%$ of the total number of conflicts. This is significant because, for a relatively large size problem instance, by simply using 4 independent memory banks and a 4-element mapping table, we effectively reduced the total memory access conflicts by almost $42 \%$, while a brutal-force method needs 259 independent memory banks to achieve conflict-free memory accesses.

\section{MATHEMATICAL FOUNDATION: GRAPH FOLDING AND COLORING}

Before describing our algorithm in detail, we mathematically define several important concepts in our methodology. Let $\mathcal{G}=(V, E)$ denote the memory access conflict graph, coloring $\mathcal{G}$ is the process of assigning each vertex $v \in V$ with a distinct color, such that no two adjacent vertices connected by an edge $e \in E$, have the same color. In mathematics, if a graph $\mathcal{G}$ can be colored with $k$ colors, the graph is termed as $k$-colorable, and the smallest $k$ for which the graph $\mathcal{G}$ is $k$-colorable is defined as the chromatic number of $\mathcal{G}$, denoted as $\chi(\mathcal{G})$. If we proceed with optimally coloring this conflict graph with $k$ colors, optimal memory banking can be readily computed to incur zero memory access conflict with the same $k$ independent memory banks. Despite of the elegance of this coloring-based scheme, unfortunately, it is well-known that even determining if a graph $\mathcal{G}$ is $k$-colorable for $k \geq 3$ proves to be NPcomplete [8]. This makes solving a realistic large-scale memory banking problem infeasible.

We now introduce the concept of graph folding. Note that our definition of graph folding deviates from the standard definition in [9]. Specifically, Let $G_{1}$ and $G_{2}$ be graphs, we define a graph map to be a discrete function $\mathcal{F}: G_{1} \longrightarrow G_{2}$ that satisfies two conditions: 1) for any vertex $v \in V\left(G_{1}\right), \mathcal{F}(v) \in V\left(G_{2}\right)$. 2) for each edge $e \in E\left(G_{1}\right), \operatorname{dim}(\mathcal{F}(e)) \leq \operatorname{dim}(e)$. Mathematically [], a graph map $\mathcal{F}$ is called a graph folding if and only if 1) $\left|V\left(G_{2}\right)\right|<$ $\left|V\left(G_{1}\right)\right|$ and for any $v_{i} \in E\left(G_{1}\right)$ and any $v_{j} \in E\left(G_{1}\right)$, the edge 

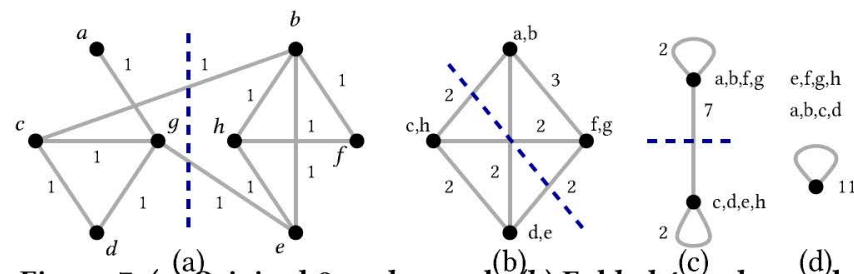

Figure 7: (a) Original 8-node graph. (b) Folded 4 -node graph (c) Folded 2-node graph. (d) Folded 1-node graph.

$\left(\mathcal{F}\left(v_{i}\right),\left(\mathcal{F}\left(v_{j}\right) \in E\left(G_{2}\right)\right.\right.$. where $V(G)$ and $E(G)$ denote the vertex set and edge set of graph $G$, respectively. To conceptually illustrate the idea of graph folding, in Fig. 7, we depict a folding process of a small irregular graph. As folding proceeds, the original 8-node and 11-edge graph is folded into a smaller and smaller graph with different edge weights. Note that the edge weight sum of the folded graph equals to the total edge number of the unfolded original graph. Moreover, self loops of nodes will be produced during the folding process, which are absent in the original unfolded graph. Finally, the folding we consider is a special case of the general definition of $\mathcal{F}$.

\section{SPECIFIC TECHNIQUES OF COMPUTING BANK INDEX AND INTRA-BANK OFFSET}

\section{Priority coloring}

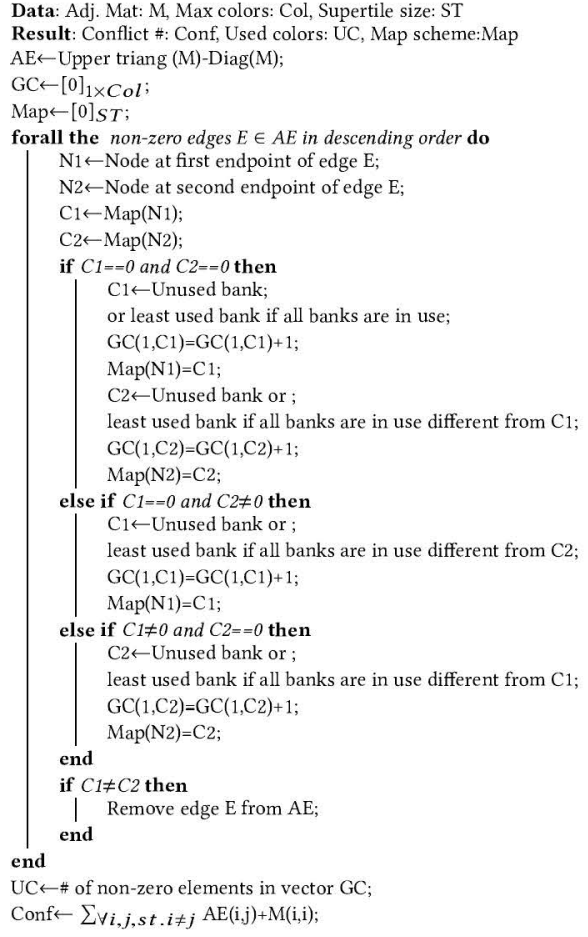

Algorithm 1: Our proposed priority weighted coloring algorithm

In graph theory, weighted coloring generalizes the well-known unweighted vertex coloring. Even for unweighted coloring, although exact algorithm exists, coloring any reasonably sized graph turns out to be computationally prohibitive (NP-complete). Not surprisingly, a optimal method for the minimum-weighted coloring problem on arbitrary graphs doesn't exist [10]. Only a few heuristic

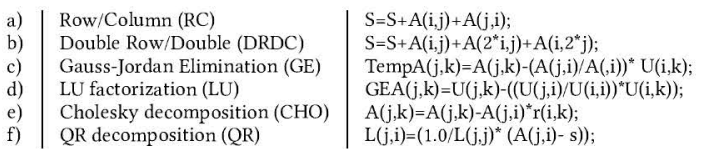

Figure 8: Six non-stencil computing kernels. Only the innermost statement is shown to save space.

approximate algorithms have been developed with the goal of finding tighter upper bounds for the maximum-weighted clique problem. In this paper, we propose a heuristic algorithm listed in Algorithm 1 to perform the prioritized color mapping for a given folded memory conflict graph, where each edge weight denotes how many memory conflicts will occur throughout all iterations. Specifically, starting from the heaviest (most repeated pair of nodes accessed together) edge to the lightest one, we try to assign distinct colors to the nodes connected by the edge in a greed fashion. Along this coloring process, we remove any edge whenever its both ends are colored. It should be clear that this algorithm will definitely terminate. The optimal ratio of this method can be analytically bounded as in [10].

\section{Computing Bank Index and Intra-Bank Offsets}

Once the folded memory conflict graph is fully colored, the bank index of each memory access can be deduced from its data array indexes. Specifically, for a $k$-dimensional problem, Bank $(\mathrm{B})=$ $\mathrm{BST}\left(x_{0} \bmod \mathrm{ST}_{0}, x_{1} \bmod \mathrm{ST}_{1}, \ldots, x_{k} \bmod \mathrm{ST}_{k}\right)$ In addition, the intra bank offset of each memory location can be calculated by determining both its intra-folded graph offset and global offset. Specifically, the intra-folded-graph offset can be calculated at compile time by scanning the folded graph and assigning all the elements in the same memory bank a distinct number. To access the intra-folded-graph offset, one accesses the address supertile (AST) in the same fashion one would to access the bank super tile. Finally, to calculate the global offset, given a memory access point in $k$-dimensional space, one just has to count the number of folded graphs in the lower dimensions and multiply it by the maximum offset of the bank per folded graph. To simplify calculations, one can consider the same maximum offset $M$ for all banks as the greatest of all offsets in the supertile. Note this might lead to some memory waste because some colors might have been used more often than other, but since the coloring algorithm tries to balance color utilization, it is expected that the intra-folded-graph offsets for all the banks to be roughly the same, the waste is expected to remain minimal. Mathematically, a memory access with coordinates $\left(x_{0}, x_{1}, \ldots, x_{k}\right)$ for a problem size $w_{0} \times w_{1} \times \cdots \times w_{k}$, and a folded graph of size $\mathrm{ST}_{0} \times \mathrm{ST}_{1} \times \cdots \times \mathrm{ST}_{k}$, with a maximum intra-foldedgraph offset of $M$, the global offset can be calculated as:

$$
\text { Global offser }(\mathrm{GO})=\mathrm{M} \cdot \sum_{i=0}^{k-1}\left(\left\lfloor\frac{x_{i}}{S T_{i}}\right\rfloor \cdot * \prod_{j=0}^{i-1}\left\lceil\frac{w_{j}}{S T_{j}}\right\rceil\right)
$$

Note for any given problem, equation this equation can be expanded at compile time given that the dimensionality of the problem does not change, thus being able to pre-compute some of the terms. Particularly all the products in $\Pi$. Finally, the computed intra-bank offset is the sum of the intra-folded-graph offset (STO) and the global offset (GO).

\section{RESULTS AND ANALYSIS}

To validate our graph-folding-based methodology, we consider six non-stencil computing kernels as listed in Fig. 8, all of which are 
widely used in scientific computing and exhibit various degrees of memory access irregularity. For each kernel, we followed the memory banking scheme outlined in Section 4 and obtained the memory banking map with three different sizes: $8 \times 8,16 \times 16$, and $32 \times 32$ and a maximum partition factor of 32 (available banks). We then synthesized these mapping functions for these different folded graph sizes and different number of available memory banks. The generated Verilog code was then compiled with Vivado HLS 2015.4 and implemented on a Kintex 7 xc7k160tffg676-3 chip.

Fig. 9 presents our experimental results for all six non-stencil computing kernels. For each kernel, we measured the conflict ratio for 4 different folded graph sizes and different number of memory banks up to 32 . Overall, for a fixed folded graph size, the more banks are available, the greater the conflict reduction. This trend continues until there are enough colors to ensure all adjacent nodes can be assigned a distinct color. The number of banks required to reach this point will vary depending on the coloring strategy implemented. A greedy approach like the one used in this paper will most likely not give the optimal coloring scheme except for the simplest of conflict graphs such as the DR and DRDC kernels. Once all inter-node edges are removed, only the self-conflicts will contribute to the total amount of conflicts. The only way to eliminate or modify these self conflicts is by changing the geometry of the folded graph. This effect can be seen by comparing the number of conflicts for a given partition factor between two supertile sizes. However, there is some exception. Most notably, the QR factorization in Fig. 9(d) incurs a much higher conflict ratio for a particular partition factor than previous, smaller ones. This is most likely due to a compound effect of the color selection priority of the coloring algorithm, the number of available colors, and the resulting structure of the conflict graph. These factors influence the order in which the nodes are colored, and the total amount of available colors determines the distribution of the colors. The algorithm tries to balance the colors used in order to achieve an uniform distribution (no color is used significantly more than any other), the effects of the greedy selection can accumulate over time leaving low-weight edges considered at the end of the algorithm connecting same-colored nodes. However, one can still observe the aforementioned conflict reduction trend.

To validate the performance benefits of our graph-based memory banking scheme, we start with inputting the memory access patterns of all test benchmarks into a Matlab script which computes the bank assignment and relative offset inside a super-tile for all memory locations. A Matlab script takes the information about the bank and offset super-tile and automatically generates new transformed code in $C$. This transformed $C$ code is then used as an input to the Vivado HLS 2016.2 from Xilinx, which generates the HDL files in Verilog. The software also automatically generates a Vivado HLx 2016.2 project with the Verilog code already included. This project is synthesized and implemented. This software suite is also the same tool used to report post implementation resource usage and power estimation. To illustrate, we have listed a transformed code snippet in Fig. 10.

Table 1 lists the resource utilization and the achievable clock period for a fully pipelined ( $\mathrm{I}=1$ ) implementation of the mapping function for the 4 accesses of the GE kernel on a problem size of elements for $1024 \times 1024$ different supertile sizes. As we can see from the table, the resource utilization does not change in any significant way when the folded graph size increases from $8 \times 8$ to

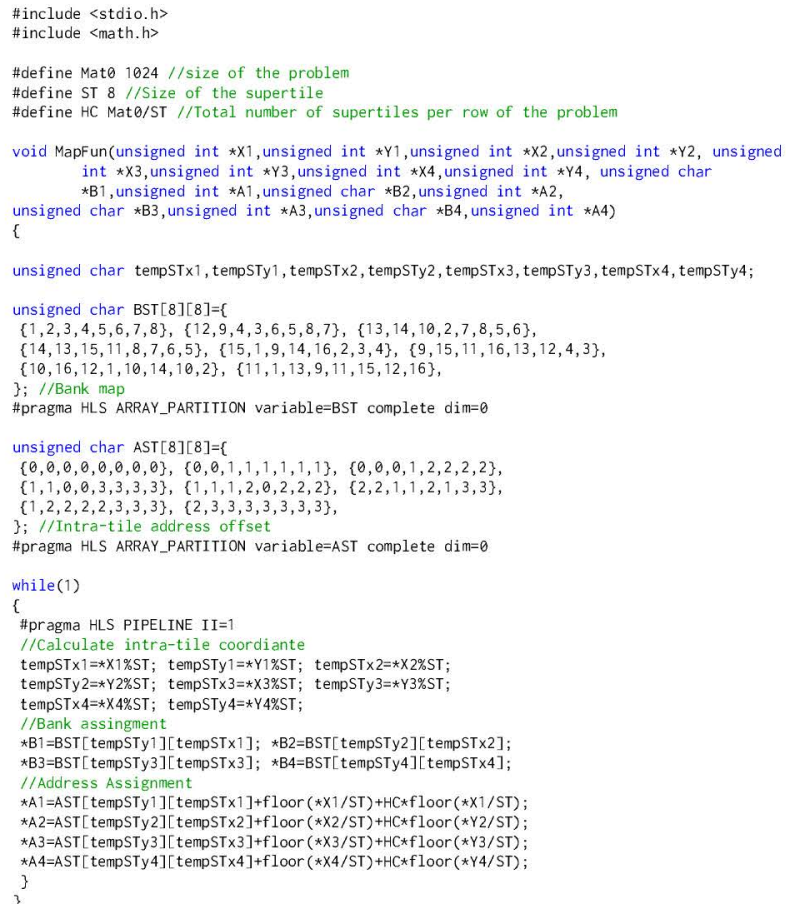

Figure 10: Code template for the mapping function of the GE with a supertile of size $8 \times 8$.

\begin{tabular}{|c|c|c|c|c|c|}
\hline ST size & Resource & Utilization & Available & $\begin{array}{c}\text { Utilization } \\
(\%)\end{array}$ & $\begin{array}{c}\text { CP } \\
(\mathrm{nS})\end{array}$ \\
\hline \multirow{4}{*}{$8 \times 8$} & LUT & 8936 & 101400 & 8.81 & \\
\cline { 2 - 5 } & LUT RAM & 1 & 35000 & 0.01 & \multirow{4}{*}{8.55} \\
\cline { 2 - 5 } & FF & 6435 & 202800 & 3.17 & \\
\cline { 2 - 5 } & BRAM & 7 & 325 & 2.15 & \\
\cline { 2 - 5 } & DSP & 68 & 600 & 11.33 & \\
\hline \multirow{4}{*}{$36 \times 16$} & LUT & 9094 & 101400 & 8.97 & \\
\cline { 2 - 5 } & LUT RAM & 1 & 35000 & 0.01 & \multirow{4}{*}{8.6} \\
\cline { 2 - 5 } & FF & 6467 & 202800 & 3.19 & \\
\cline { 2 - 5 } & BRAM & 7 & 325 & 2.15 & \\
\cline { 2 - 5 } & DSP & 68 & 600 & 11.33 & \\
\hline & LUT & 9698 & 101400 & 9.56 & \\
\cline { 2 - 5 } & LUT RAM & 1 & 35000 & 0.01 & \\
\cline { 2 - 5 } & FF & 6480 & 202800 & 3.2 & \\
\cline { 2 - 5 } & BRAM & 7 & 325 & 2.15 & \\
\cline { 2 - 5 } & DSP & 68 & 600 & 11.33 & \\
\hline
\end{tabular}

Table 1: Resource utilization for the GE kernel, problem size $1024 \times 1024$, and different supertile sizes.

$32 \times 32$. Another observation is that the bulk of the mapping hardware was likely performed by this BRAM, and since the number of IO remained constant, the small difference in the LUT and FF is due to the maximum intra bank address, which reaches a higher value for bigger folded graphs.

\section{RELATED WORK}

In HLS, work on ensuring parallel memory access in irregular or amorphous kernels is relatively scarce. Recently, a new approach [5] uses run-time memory traces and their associated bits to generate an optimal bit mask, stored as a multi-level lookup table, to minimize memory conflicts. The main advantage of this method, contrary to [2] and [3], is that it can in theory be used for any kind of kernel, stencil or not, with better results than [6] for non-stencils 


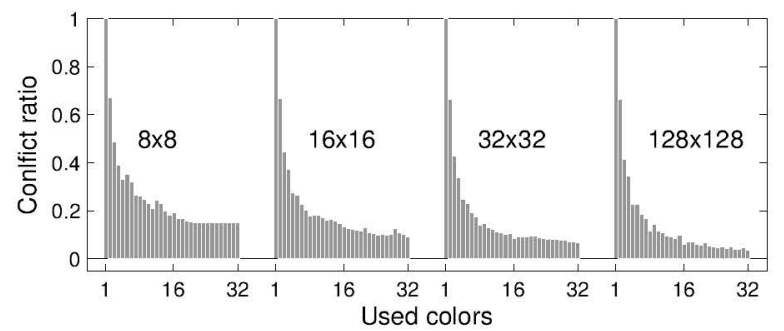

(a) GE Kernel

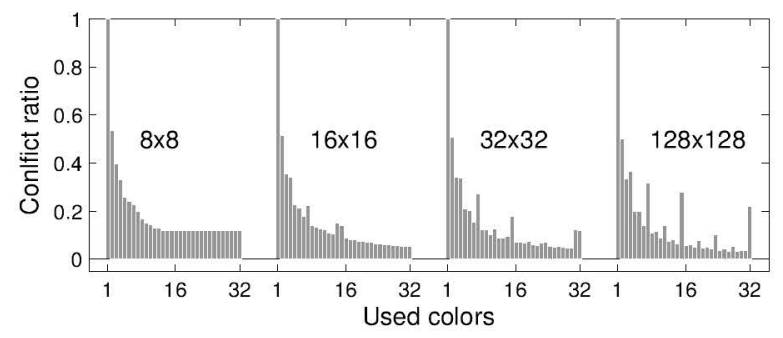

(c) CHO Kernel

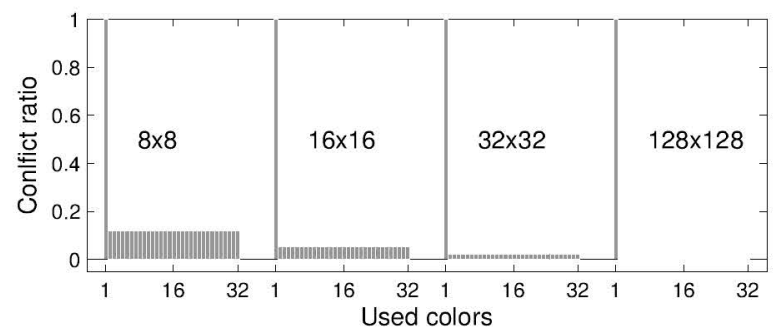

(e) RC Kernel

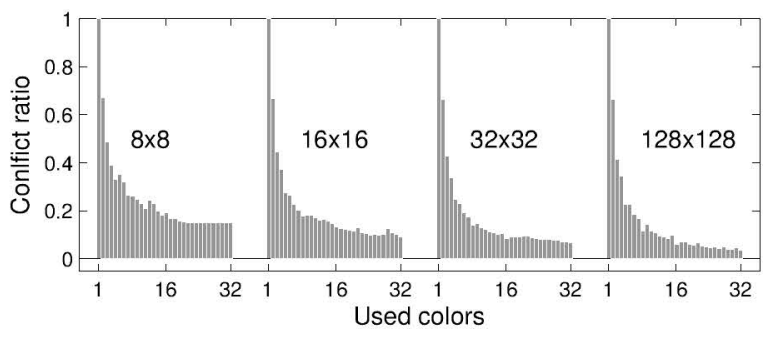

(b) LU Kernel

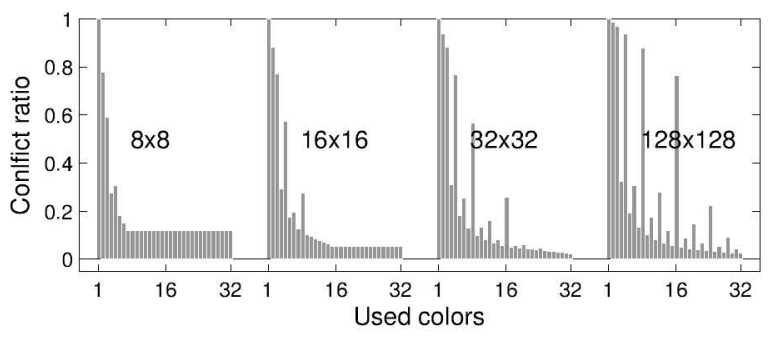

(d) QR Kernel

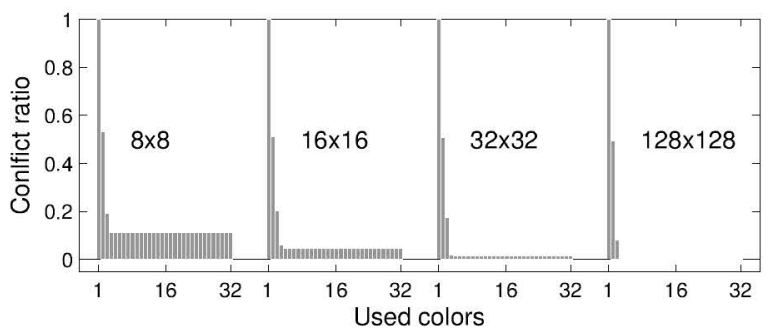

(f) DRDC Kernel

Figure 9: Conflict ratio for various non-stencil kernels for various folding sizes: $8 \times 8,16 \times 16,32, \times 32$, and 128x128

and no worse for stencils. Its disadvantage is the total run time needed to test all possible masks to find the best with no guarantee on the upper limit needed to achieve an optimal solution. More recently, the Polytope Emptiness Test (PET) method presented in [1] can solve imperfectly nested loops and some cases when the offset vectors are 0 's or even the same, but the polytope will be nonempty if at some point two or more accesses try to access the same memory point at the same time, which will be considered a conflict. Another limitation of the PET method is that it does a heuristic search for both the bank number and the alpha that sets the linear partition, although a new way of computing " $\alpha$ " has been found in [4]. Still, this method requires perfectly nested loops, can not determine suitable memory block size, and only tackles a single statement. In contrast, our proposed method is quite general, i.e., imposing no requirement on the shape and regularity of stencils, and solely relies on graph analysis, therefore computationally efficient to perform.

\section{CONCLUSIONS}

To perform effective memory banking in HLS, we have presented an intuitive scheme based on priority coloring a weighted graph generated by strategically folding a fundamental conflict graph. This work extends many other memory banking schemes that can only effectively tackle stencil-based computing kernels, thus being applicable to any kind of kernel code with even irregular memory access patterns as long as they are static.

\section{REFERENCES}

[1] Y. Wang, P. Li, and J. Cong, "Theory and algorithm for generalized memory partitioning in high-level synthesis," in Proceedings of the 2014 ACM/SIGDA International Symposium on Field-programmable Gate Arrays, FPGA '14, (New York, NY, USA), pp. 199-208, ACM, 2014.

[2] A. Cilardo and L. Gallo, "Improving multibank memory access parallelism with lattice-based partitioning," ACM Trans. Archit. Code Optim., vol. 11, pp. 45:1$45: 25,2015$.

[3] J. Escobedo and M. Lin, "Tessellating memory space for parallel access," in ASPDAC, 2017.

[4] C. Meng, S. Yin, P. Ouyang, L. Liu, and S. Wei, "Efficient memory partitioning for parallel data access in multidimensional arrays," in 2015 52nd ACM/EDAC/IEEE Design Automation Conference (DAC), pp. 1-6, June 2015.

[5] Y. Zhou, K. M. Al-Hawaj, and Z. Zhang, "A new approach to automatic memory banking using trace-based address mining," in Proceedings of the 2017 ACM/SIGDA International Symposium on Field-Programmable Gate Arrays, FPGA '17, (New York, NY, USA), pp. 179-188, ACM, 2017.

[6] Y. Wang, P. Li, and J. Cong, "Theory and algorithm for generalized memory partitioning in high-level synthesis," in Proceedings of the 2014 ACM/SIGDA International Symposium on FPGA, pp. 199-208, 2014.

[7] N. Christofides, "An algorithm for the chromatic number of a graph," The computer journal, vol. 14 (1), pp. 38-39, 1971.

[8] A. Wigderson, "Improving the performance guarantee for approximate graph coloring," Journal of the Association for Computing Machinery, vol. 30, pp. 729$735,1983$.

[9] E.-K. E and E.-E. A, "Graph folding of some special graphs," Journal of Mathematics and Statistics, vol. 1, 012005.

[10] J. Xue, "Solving the minimum weighted integer coloring problem," Comput. Optim. Appl., vol. 11, pp. 53-64, Oct. 1998 